\title{
Deficit of corpus callosum axons, reduced axon diameter and decreased area are markers of abnormal development of interhemispheric connections in autistic subjects
}

\author{
Jarek Wegiel ${ }^{1 *}$ (i), Wojciech Kaczmarskil, Michael Flory ${ }^{2}$, Veronica Martinez-Cerdeno ${ }^{3}$, Thomas Wisniewski ${ }^{4}$, \\ Krzysztof Nowicki ${ }^{1}$, Izabela Kuchna ${ }^{1}$ and Jerzy Wegiel ${ }^{1}$
}

\begin{abstract}
Introduction: In autism spectrum disorder, lack of coherence and of complex information processing, and narrowly focused interests and repetitive behaviors are considered a sign of long-range underconnectivity and short-range overconnectivity. The goal of this morphometric study of five anatomically and functionally different segments of the corpus callosum (CC) was to establish patterns of differences between long-range interhemispheric connections in nine neurotypical and nine autistic subjects.

Results: Electron microscopy revealed a significant reduction in average axon diameter and axon cross-sectional area in autistic subjects, and reduction in CC segment-specific diversification of connections of functionally different cortical regions. The study shows an increase in the percentage of small diameter axons $(<0.651 \mu \mathrm{m})$ and a decrease in the percentage of axons with large diameter $(>1.051 \mu \mathrm{m})$. The total number of small-diameter axons is reduced in segment I and III by $43 \%$ on average. The number of medium- and large-diameter axons is reduced in all five CC segments by an average of 49 and $72 \%$, respectively.

Conclusions: The detected pattern of pathology suggests a failure of mechanisms controlling guidance of axons during development leading to axonal deficit, and failure of mechanisms controlling axon structure. A reduction in axon diameter may affect the velocity and volume of signal transmission, and distort functional specialization of CC segments. Significant deficits in axon number and reduction in axon size in all five CC segments appear to be substantial components of brain connectome integrity distortion which may contribute to the autism phenotype.
\end{abstract}

Keywords: Autism, Neuropathology, Corpus callosum, Axon, Electron microscopy, Morphometry

\section{Introduction}

Developmental abnormalities of brain connectivity in autism Autism spectrum disorders (ASDs) are debilitating neurodevelopmental conditions characterized by impairments in social interactions and communication combined with restricted interests and repetitive behaviors [3]. Despite

\footnotetext{
* Correspondence: Jarek.Wegiel@opwdd.ny.gov

${ }^{1}$ Department of Developmental Neurobiology, New York State Institute for Basic Research in Developmental Disabilities, 1050 Forest Hill Road, Staten Island, NY 10314, USA

Full list of author information is available at the end of the article
}

the genetic and clinical heterogeneity of ASDs, Geschwind and Levitt [16] hypothesized that a common feature of the brain across the many forms of autism is developmental disconnection, resulting from the failure to develop normal connections between higher-order association areas of the temporal, parietal, and frontal cortices. Just et al. [28] formulated an underconnectivity theory, whereas Belmonte et al. [6] suggested a combination of reduced long-distance, and increased local connectivity in ASD. Functional $[27,30,34]$ and structural $[5,31]$ neuroimaging findings support the concept of disconnection $[12,15]$

(c) The Author(s). 2018 Open Access This article is distributed under the terms of the Creative Commons Attribution 4.0 International License (http://creativecommons.org/licenses/by/4.0/), which permits unrestricted use, distribution, and reproduction in any medium, provided you give appropriate credit to the original author(s) and the source, provide a link to the Creative Commons license, and indicate if changes were made. The Creative Commons Public Domain Dedication waiver (http://creativecommons.org/publicdomain/zero/1.0/) applies to the data made available in this article, unless otherwise stated. 
and underconnectivity $[25,28]$. The first postmortem study of axonal connections in autism published by Zikopoulos and Barbas [61] revealed disconnection of long-distance pathways and excessive connections between neighboring areas in the white matter adjacent to the anterior cingulate cortex, orbitofrontal cortex and lateral prefrontal cortex suggesting contribution of these developmental defects to diagnostic behavioral alterations in autism. The combined effect of developmental abnormalities reported in imaging and postmortem studies is loss of integrity in brain connectivity, which appears to be essential for deficits of higher-order social, emotional, and communication activities in autistic subjects $[46,61]$.

Corpus callosum (CC) developmental abnormalities in autism The CC, the largest fiber tract in the human brain $[23,45,55]$, is a primary target for testing the hypothesis of long-range underconnectivity. The CC connects brain hemispheres and facilitates integration of sensory and motor information with executive functions, social interactions, and language [38]. Structural MRI of the brain of autistic subjects shows a thinner CC and reduced CC midsagittal area, suggesting a deficit of long-range interhemispheric axonal connections [9, 21, 27, 36, 41, 42]. Brain fiber tractography based on diffusion tensor imaging divided the human $\mathrm{CC}$ into five segments, with axons projected through the brain midline by neurons of the prefrontal (S I), premotor and supplementary motor (S II), motor (S III), sensory (S IV), and parietal, occipital, and temporal cortices (S V) [24]. Morphometry of the CC of neurotypical subjects reveals a segment-specific ratio of axons of different diameter. In the brains of control subjects, the percentage of small-diameter axons is the largest in S1 and decreases in posterior segments, whereas the percentage of medium- and large-diameter axons increases in the middle and caudal portions of the CC [45]. Axon diameter, cross-sectional area, and myelin sheath thickness correlate with the velocity and volume of signal transmission [39, 47, 48]. Therefore, a CC segment-specific combination of these structural characteristics reflects the function of axons connecting specialized cortical regions.

To explain clinical MRI characterization of developmental anomalies of interhemispheric connections, we performed the first postmortem morphometric light microscopy study of the CC of autistic individuals [56]. The study revealed a significant deficit in interhemispheric axonal connections and two types of developmental abnormalities contributing to this deficit: a topographically selective partial CC agenesis and topographically non-selective hypoplasia. The detected regional agenesis and hypoplasia in CC segments are signs of focal interhemispheric disconnection and diffuse underconnectivity in autism.

\section{CC segmentation}

There are no macroscopic anatomical landmarks that delineate distinct callosal areas in a midsaggital CC cross-section of the human corpus callosum. Several geometric partitioning schemes have been designed including the commonly used Witelson [60] CC division into five vertical segments. However, this segmentation was based predominantly on non-human primate studies. In this study, Hofer and Frahm [24] CC segmentation based on human brain diffusion tensor imaging (DTI) was applied. DTI fiber tracking identifies axonal bundles that cross the $\mathrm{CC}$ midline and separates transcallosal fiber tracts with respect to their specific cortical projections. Hofer and Frahm classification distinguishes segment (S) I with prefrontal cortex projections, SII with premotor and supplementary connections, SIII with motor connections, SIV with sensory cortex connections, and SV with parietal, temporal and occipital connections. Segmentation of the CC from the most rostral to the most caudal end of the $\mathrm{CC}$ defines the rostro-caudal extent of each segment with SI - 17\%; SII $33 \%$, SIII - 17\%; SIV -8\%; and SV - 25\%.

\section{Aims}

The aims of this electron microscopy study of axons in all five structurally and functionally different segments in the $\mathrm{CC}$ of autistic and control subjects were (a) to define the range of deficits in the number of small-, medium-, and large-diameter axons in CC segments connecting functionally diverse cortical regions; and (b) to define distortion in the segment-specific ratio of axons of different diameters as a marker of loss of specialization of interhemispheric connectivity in autism.

\section{Materials and methods \\ Cohort}

This study of the corpus callosum in autistic subjects is a component of a project designed by several research groups and supported by Autism Speaks and the Autism Tissue Program. The overall goal was to overcome problems associated with scarcity of material for postmortem studies resulting in studies of 3-4 cases by different groups applying different clinical criteria and different methods of examination of a few brain regions. Screening of 18 pairs of autistic and control subjects identified 12 age- and sex-matched pairs corresponding to inclusion criteria. One brain hemisphere of these subjects was processed and embedded in celloidin for detection of global patterns of anatomical, cellular and subcellular changes in autism.

This electron microscopic study of the CC was performed on 9 pairs of autistic and control subjects with well-preserved CC ultrastructure. The demographics, information about intellectual deficits, seizures, and 
cause of death are summarized in Table 1 . In both the autistic and control group, the same number of males (6) and females (3) was examined. The age of subjects with autism was 5 to 60 years (25.4 on average) and of control subjects 4 to 52 years (26.3 on average). The diagnosis of autism was confirmed with the Autism Diagnostic Interview, Revised [33]. Intellectual deficit ranging from mild to severe was reported in six autistic subjects, and seizures in five cases. The average postmortem interval (PMI) was 21.2 and $15 \mathrm{~h}$ in the autistic and control groups, respectively. Differences in age, PMI, brain weight, and brain weight loss during dehydration were not significant.

\section{Tissue sampling for estimation of CC area, number of axons, and EM-based axon morphometry}

The brain hemisphere was fixed in $10 \%$ buffered formalin for at least 3 months, washed for $24 \mathrm{~h}$ in water to remove fixer, dehydrated in increasing concentrations of $\mathrm{ETOH}$, embedded in celloidin [22] and cut into $200-\mu \mathrm{m}$-thick frontal hemispheric sections. For morphometric study, a 2-mm wide CC strip was cut off from every twelfth coronal hemispheric section, impregnated with $2 \%$ osmium tetroxide, dehydrated and embedded in Epon. Resin blocks were cut into 1 - $\mu$ m-thick sections. Samples were oriented to cut axons perpendicularly to axon long axis and stained with a $2 \%$ solution of p-phenylenediamine (PPD). Every 60th section was used to determine the entire midsagittal area of the $\mathrm{CC}$ and each of the five segments, whereas 21 equidistant sections were used for estimation of the total number and numerical density of axons under a light microscope. The results were summarized in our previous paper [56].

\section{Electron microscopy study}

Thirteen Epon blocks represented the entire rostro-caudal extent of the $\mathrm{CC}$ in each brain of nine control subjects and nine autistic subjects, including three Epon blocks for S I, S II, and S V and two blocks for the shorter segments, III and IV (Fig. 1). For electron microscopy, Epon blocks were cut into 60-nm-thick sections using a Reichert Ultracut S (Leica, Austria) ultramicrotome and Diatome $45^{\circ}$ knife (Diatome, U.S., Fort Washington, PA, USA), and collected on formvar-coated copper grids. Sections were stained with uranyl acetate and photographed at a magnification of 15,000x using a Hitachi H7500 transmission electron microscope with a CCD camera and Advanced Microscopy Techniques' (AMT) Image Capture Engine (Danvers, MA). For each case, 12 electron micrographs were used for measurements of axon diameter, cross-sectional area, and myelin thickness. Background

Table 1 Preserved tissue and clinical and postmortem records

\begin{tabular}{|c|c|c|c|c|c|c|c|c|}
\hline Group and case ID & Sex & Age $(y)$ & Intellectual deficit & Seizures age of onset & Cause of death & PMI (h) & BW (g) & Weight loss (\%) \\
\hline$\overline{\mathrm{A} 1}$ & $\mathrm{~F}$ & 5 & - & - & Traumatic injuries & 13 & 1390 & 52 \\
\hline$A 2$ & M & 8 & - & - & Rhabdomysarcoma & 22 & 1570 & 45 \\
\hline A3 & $\mathrm{F}$ & 11 & Mild & $4.5 \mathrm{~m}$ & Seizure-related drowning & 13 & 1460 & 52 \\
\hline A4 & M & 13 & Severe & $2 y$ & Seizure-related & 8 & 1470 & 39 \\
\hline A5 & $\mathrm{F}$ & 21 & Moderate & $5 y$ & Seizure related resp. failure & 50 & 1108 & 43 \\
\hline A6 & M & 23 & Severe & $23 y$ & Seizure-related resp. failure & 14 & 1610 & 60 \\
\hline A7 & M & 36 & Severe & - & Cardiac arrest & 24 & 1480 & 44 \\
\hline A8 & M & 52 & - & - & Heart failure & 11 & 1324 & 53 \\
\hline A9 & M & 60 & Moderate & $3 y$ & Pancreatic cancer & 26 & 1210 & 38 \\
\hline Mean \pm SD & & $25.4 \pm 19.8$ & & & & $21.2 \pm 13.6$ & $1402 \pm 164$ & $47.3 \pm 7.3$ \\
\hline C1 & $\mathrm{F}$ & 4 & - & - & Acute bronchopneumonia & 17 & 1530 & 49 \\
\hline C2 & M & 14 & - & - & Electrocution & 20 & 1464 & 44 \\
\hline $\mathrm{C} 3$ & $\mathrm{~F}$ & 15 & - & - & Traumatic injuries & 9 & 1250 & 49 \\
\hline C4 & $\mathrm{F}$ & 20 & - & - & Traumatic injuries & 9 & 1340 & 52 \\
\hline C5 & M & 23 & - & - & Ruptured spleen & 6 & 1520 & 41 \\
\hline C6 & M & 29 & - & - & Traumatic injuries & 13 & 1514 & 49 \\
\hline C7 & M & 32 & - & - & Asphyxia & 24 & 1364 & 42 \\
\hline $\mathrm{C} 8$ & M & 48 & - & - & Heart atherosclerosis & 24 & 1412 & 39 \\
\hline C9 & M & 52 & - & - & Heart atherosclerosis & 13 & 1430 & 48 \\
\hline Mean \pm SD & & $26.3 \pm 15.8$ & & & & $15 \pm 6.6$ & $1424 \pm 94$ & $45.9 \pm 4.5$ \\
\hline
\end{tabular}

$A$ autism, $C$ control, PMI postmortem interval, $h$ hours; Weight loss, decrease (\%) of hemispheric brain sample weight during dehydration in ethyl alcohol; $S D$ standard deviation 


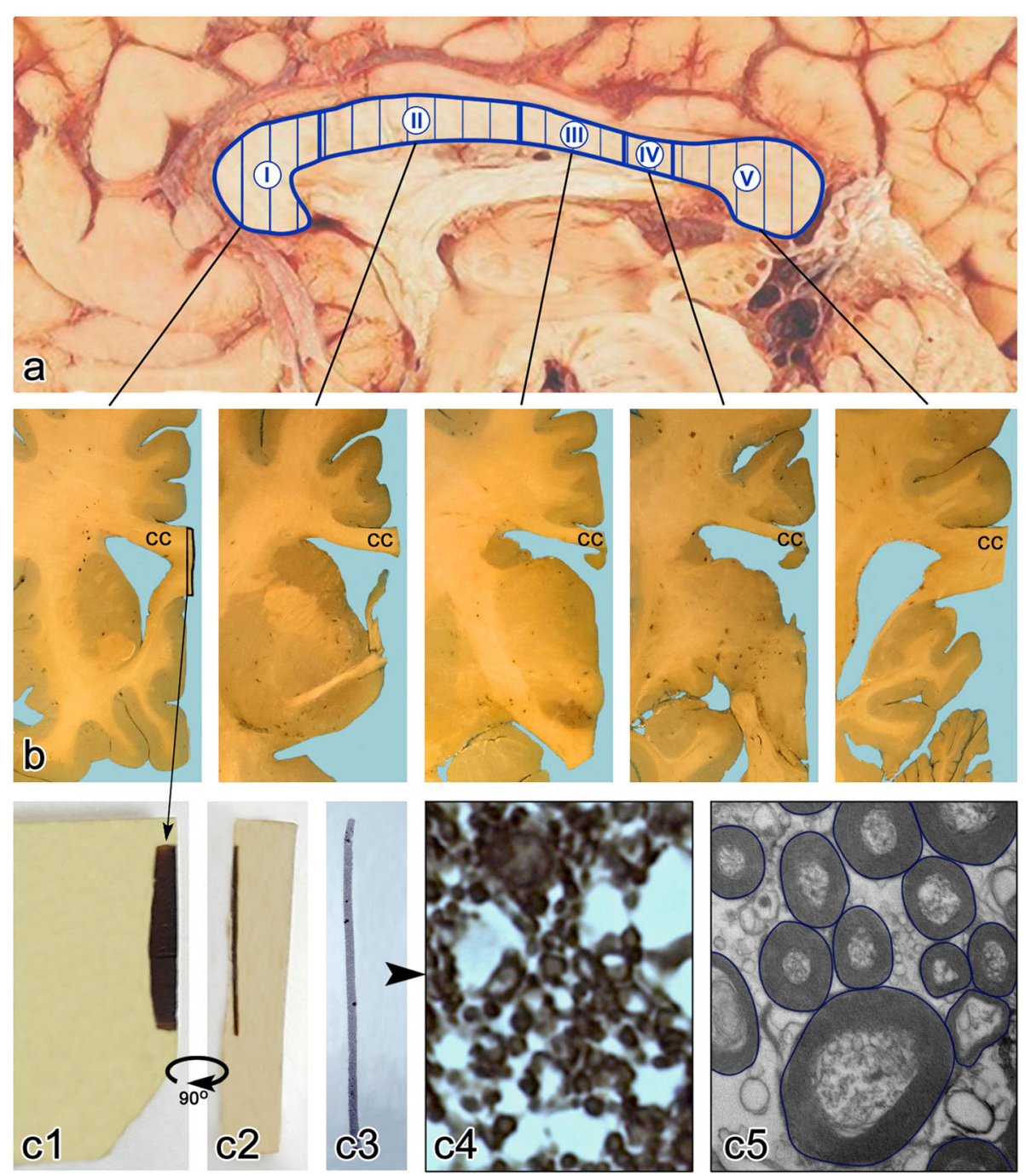

Fig. 1 CC sampling for light and electron microscopy study. Diffusion tensor imaging and fiber tractography-based vertical partitioning of the CC into five segments corresponding to interhemispheric cortical connections (a). Celloidin embedded brain hemisphere was cut into $200-\mu m$-thick gapless serial sections (b). From thirteen equidistant CC samples representing all segments (b) a 2-mm-wide CC sample was cut off and embedded in Epon (c1). Semithin sections were cut (c2) and stained with PPD (c3) for light microscopy morphometric studies [56]. For the EM morphometric study of axons Epon blocks were trimmed and 60-nm-thick sections were cut and stained with uranyl acetate. The calibrated electron micrographs obtained at 15,000x magnification were used for manual delineation of the axon border (c4) to estimate the diameter and crossectional area of the axon, as well as to estimate the thickness of the myelin sheath measured at four equidistant points. The measurements were performed with Image J software

correction was applied to reduce risk of distortions during image analysis. For morphometric analysis, the images were saved in the jpeg format.

\section{Axon measurements}

The study of CC axons was limited to myelinated axons, which are more common (comprising approximately $90 \%$ of all axons [32] and are better preserved than non-myelinated axons [45]. On average in the CC of each control and autistic subject, 1676 and 1486 axons, respectively, were measured. The total number of axons measured in nine control subjects was 15,085, and in nine autistic subjects 13,376 . Oversampling was necessary to detect significant differences in all CC segments, including the smallest sector IV representing only $8 \%$ of the linear rostro-caudal CC extent [24]. Axons were measured manually by delineation of the outer axon myelin contour (Fig. 1). Image J software provided measurements of axon diameter (Feret's diameter, $\mu \mathrm{m}$ ) and area $\left(\mu \mathrm{m}^{2}\right)$. Average myelin thickness of axons was estimated by measuring the myelin sheath thickness at four equidistant points. 


\section{Statistical analysis}

Analyses of axonal diameter, cross-sectional area, and myelin thickness across diagnostic groups and segments of the $\mathrm{CC}$ were done in repeated-measures ANOVAs, using the conservative Greenhouse-Geisser adjustment for the non-sphericity of the dependent variables across segments [19]. Comparisons of percentages of small-, medium-, and large-diameter axons across five segments were done using the wsanova routine [17]. Post-hoc analyses used one-way ANOVAs and T-tests. All analyses were performed using Stata version 15.1 [51]. To address the potential increase in the likelihood of Type II errors (false positives) with multiple comparisons, we have used control of the False Discovery Rate (FDR) [7] rather than performing Bonferroni-type corrections of our criterion for significance [18].

\section{Results}

\section{Differences between distribution of axon diameter in} control and autistic subjects

Characterization of axon diameter distribution was based on comparison of an average of 335 axons per segment per control subject and 297 axons per segment per autistic subject. The reason for examination of fewer axons in the autistic group was lack of axons in S IV and a partial deficit in the caudal part of S III and the anterior part of S V in three autistic subjects with partial CC agenesis.

The distribution curves for all five segments (Fig. 2) suggested that three intervals $(<0.651 \mu \mathrm{m} ; 0.651-$ $1.051 \mu \mathrm{m}$ and $>1.051 \mu \mathrm{m}$ ) identify differences in the prevalence of small-, medium-, and large-diameter axons. This classification was applied to compare the percentage of small-, medium-, and large-diameter axons in control and autistic groups. The axon diameter distribution curves for each of the five CC segments revealed an increased percentage of small-diameter axons and a decreased percentage of large-diameter axons in autistic subjects. The prevalence of small- and the deficit of large-diameter axons was relatively low in S I, but the difference became larger in the four posterior segments.

The detected differences among autistic subjects in the ratio of varying diameter axons in the CC segments reflected a distortion in structural and functional specialization of individual CC segments connecting functionally different cortical regions. To define interhemispheric connection abnormalities, inter-segmental differences in average axon diameter, cross-sectional area and myelin thickness, and the percentage and total number of small-, medium-, and large-diameter axons were characterized within the control and autistic groups.
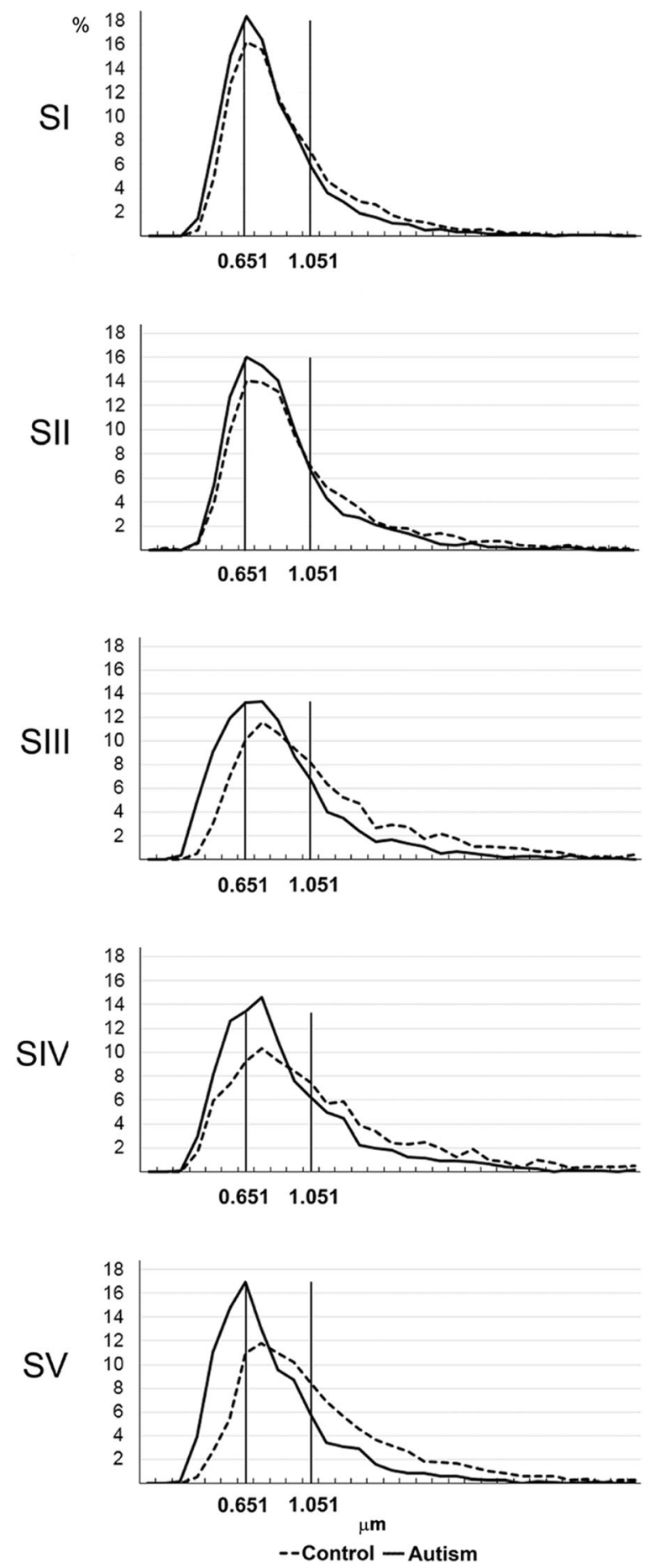

Fig. 2 Differences between axon diameter distribution in autism and control. The distribution curves of axon diameter in all five CC segments in autistic and control subjects revealed a higher percentage of small-diameter axons and a lower percentage of large-diameter axons in autistic subjects, with more prominent differences in posterior S III- S V than in anterior S I and S II 
Differences in average axon diameter, cross-sectional area and myelin thickness in CC segments in neurotypical control and autistic subjects

An overall ANOVA analysis of axon measurements showed that in the autistic subjects, average axon diameter was significantly less than in the control group (F [3] = 18.31, $p=0.0001$ ) and diameter differed across segments $(\mathrm{F}[6]=7.32$, Greenhouse-Geisser $p=0.0012)$ [19].

Within-segment ANOVA-based comparison of average axon diameter in autistic and control subjects revealed a significantly smaller diameter in autistic subjects, with the smallest difference in S I (11.2\%) and a linear increase to a $28.6 \%$ deficit in S V (Table 2, Fig. 3). Reduced axon diameter is paralleled by a very significant deficit in axon cross-sectional area ranging from $24.6 \%$ in $\mathrm{S} 1$ to $51.6 \%$ in $\mathrm{SV}$ in autistic subjects. Measurements of the thickness of the axon myelin sheath revealed insignificant deficits in S I-IV and a significant $15.8 \%(p<0.018)$ deficit limited to SV.

The second analysis, focused on detection of differences between axon diameters in individual segments of the $\mathrm{CC}$ in neurotypical control subjects, revealed significant intersegmental differences, with an increase of average axon diameter from $0.869 \mu \mathrm{m}$ in S I to $1.108 \mu \mathrm{m}$ in S III, $1.120 \mu \mathrm{m}$ in S IV, and $1.075 \mu \mathrm{m}$ in S V (Fig. 3, Table 2). Analysis showed significant increase of axon diameter in three posterior segments of control subjects (Table 3). The average axon area increased from $0.613 \mathrm{um}^{2}$ in $\mathrm{S}$ I to $1.157 \mathrm{um}^{2}$ in $\mathrm{S}$ IV and $0.968 \mu \mathrm{m}^{2}$ in $\mathrm{SV}$, and the increase was significant in these segments. The increase in axon myelin thickness was small and was significant only in S III.
This pattern of significant inter-segmental differences reflects a specialization in CC connections of functionally different cortices in the human brain.

In contrast to the broad range of intersegmental differences in the control group, in autistic subjects axon diameter and area varied in a narrow range, and differences between segments were not significant (Table 3). The flat trend line reflecting a rather uniform diameter of axons and axon area in all segments in autistic subjects (Fig. 3) was a sign of lack of structural differentiation of axons in CC segments.

Autism was a less significant factor in myelin thickness than it was in diameter or area ( $\mathrm{F}[3]=5.42, p=0.0233$ ), while neither the segment nor its interaction with the diagnosis had a significant effect.

\section{Decrease in the percentage of small-diameter axons and increase in percentage of large-diameter axons in posterior segments of the CC in neurotypical control subjects}

To learn which factors define the higher, segment-specific increase in average axon diameter in S II-V of control subjects, the percentage of small-, medium-, and large-diameter axons was determined in the five segments. The percentages of axons falling within each size range differed strongly by segment for controls $(\mathrm{F}[6]=6.61$, Greenhouse-Geisser $p=0.0005$ ).

In control subjects, the percentage of small-diameter axons $(<0.651 \mu \mathrm{m})$ decreased from $34.6 \%$ in $\mathrm{S}$ I to $19.1 \%$ in S V $(p<0.002)$ (Tables 4,5 ; Fig. 4). The opposite trajectory was detected for large-diameter $(>1.05 \mu \mathrm{m})$ axons, with an increase in the percentage of these axons from

Table 2 The difference in average axon diameter, midsagittal area, and myelin thickness in five CC segments in autistic (A) and control (C) subjects

\begin{tabular}{|c|c|c|c|c|c|c|}
\hline \multirow[t]{2}{*}{ Parameter } & \multirow[t]{2}{*}{ Group } & \multicolumn{5}{|l|}{ CC Segment } \\
\hline & & SI & $S \|$ & $\mathrm{SIII}$ & S IV & SV \\
\hline \multirow[t]{4}{*}{ Axon diameter $(\mu \mathrm{m})(\mathrm{SE})$} & C & $0.869(0.025)$ & $0.945(0.026)$ & $1.108(0.036)$ & $1.120(0.058)$ & $1.075(0.043)$ \\
\hline & A & $0.772(0.016)$ & $0.836(0.020)$ & $0.837(0.059)$ & $0.858(0.061)$ & $0.768(0.046)$ \\
\hline & $p<$ & $0.005^{* *}$ & $0.004^{* *}$ & $0.001^{* * *}$ & $0.010^{* *}$ & $0.0002^{* * *}$ \\
\hline & Difference & $-11.2 \%$ & $-11.5 \%$ & $-24.4 \%$ & $-23.4 \%$ & $-28.6 \%$ \\
\hline \multirow[t]{4}{*}{ Axon area $\left(\mu m^{2}\right)(S E)$} & C & $0.613(0.042)$ & $0.739(0.039)$ & $1.075(0.068)$ & $1.157(0.117)$ & $0.968(0.086)$ \\
\hline & A & $0.462(0.024)$ & $0.543(0.032)$ & $0.594(0.071)$ & $0.620(0.081)$ & $0.469(0.056)$ \\
\hline & $p<$ & $0.006^{* *}$ & $0.001^{* * *}$ & $0.0002^{* * *}$ & $0.005^{* *}$ & $0.0002^{* * *}$ \\
\hline & Difference & $-24.6 \%$ & $-26.5 \%$ & $-44.8 \%$ & $-46.9 \%$ & $-51.6 \%$ \\
\hline \multirow[t]{4}{*}{ Myelin thickness $(\mu \mathrm{m})(\mathrm{SE})$} & C & $0.149(0.006)$ & $0.153(0.006)$ & $0.162(0.005)$ & $0.163(0.008)$ & $0.157(0.004)$ \\
\hline & A & $0.139(0.003)$ & $0.149(0.003)$ & $0.142(0.011)$ & $0.145(0.007)$ & $0.132(0.008)$ \\
\hline & $p<$ & 0.197 & 0.538 & 0.092 & 0.141 & $0.018^{*}$ \\
\hline & Difference & ns & ns & ns & ns & $-15.8 \%$ \\
\hline
\end{tabular}

CC Corpus callosum, SE standard error, ns not significant

${ }^{*} p \leq 0.05 ;{ }^{* *} p \leq 0.01 ;{ }^{* * *} p \leq 0.001$ 

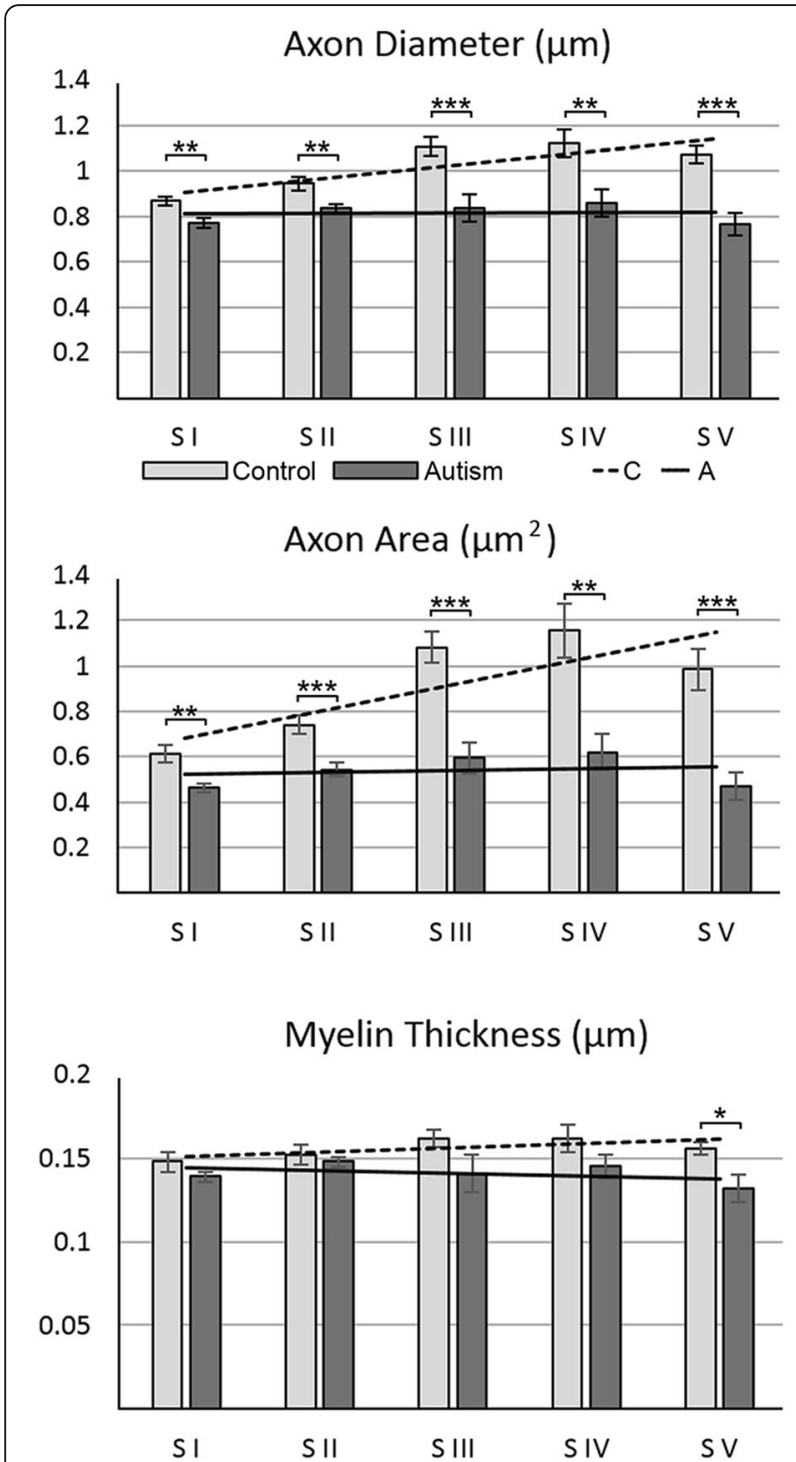

Fig. 3 Axon diameter, cross-sectional area, and myelin thickness. In the CC of control subjects, axon diameter and cross-sectional area were the lowest in $\mathrm{S} 1$ but increased significantly in $\mathrm{S} \|-\mathrm{V}$, and the increment in segments varied in a broad range. In autistic subjects, the average axon diameter and cross-sectional area were significantly less than in the control group, and differences between segments became insignificant. In contrast to variations of axon diameter and cross-sectional area, the average thickness of myelin sheath was comparable in five segments of control subjects. In autistic subjects, significant reduction in myelin thickness was detectable only in S V

$22.2 \%$ in S I to $40.2 \%$ in S IV $(p<0.0015)$. In contrast to the distinct trajectories of small- and large-diameter axons, the percentage of medium-diameter axons varied in a narrow range from $35.4 \%$ (S IV) to $43.8 \%$ (S II). These differences were not significant, and there was no detectable trend in the rostro-caudal extent of the CC (Table 5).
Table 3 Significance of difference of axon diameter, crosssectional area, and myelin thickness between $\mathrm{SI}$ and other segments in the CC in autistic and control subjects (test for diversity of axons within segments)

\begin{tabular}{llllll}
\hline Parameter & Group & S I/S II & S I/S III & S I/S IV & S I/S V \\
\hline Axon diameter & $\mathrm{C}$ & 0.055 & $0.0001^{* * *}$ & $0.003^{* *}$ & $0.0004^{* * *}$ \\
Axon cross-sectional area & $\mathrm{C}$ & $0.049^{*}$ & $0.0004^{* * *}$ & $0.003^{* *}$ & $0.0014^{* * *}$ \\
Myelin sheath thickness & $\mathrm{C}$ & 0.483 & $0.042^{*}$ & 0.101 & 0.200 \\
Axon diameter & $\mathrm{A}$ & 0.218 & 0.440 & 0.440 & 0.947 \\
Axon cross-sectional area & $\mathrm{A}$ & 0.174 & 0.174 & 0.174 & 0.931 \\
Myelin sheath thickness & $\mathrm{A}$ & 0.055 & 0.964 & 0.929 & 0.891 \\
\hline
\end{tabular}

$P$ values incorporate multiple-comparison adjustments to maintain a false discovery rate (FDR) of 0.05 . ${ }^{*} p \leq 0.05 ;{ }^{* *} p \leq 0.01$; ${ }^{* * *} p \leq 0.001$. S Segment, $C C$ corpus callosum, $C$ control, $A$ autism

Reverse trajectory in the CC of autistic subjects with an increase in the percentage of small-diameter axons and decrease of large-diameter axons in the posterior CC segments (Fig. 4, Tables 4, 5)

A comparison of the percentage of small-diameter axons in control and autistic subjects revealed an increased contribution of small-diameter axons in all segments of autistic subjects, with a significant increase limited to S I $(+24 \%$; $(p<0.027)$ and S V $(+143 \% ; p<0.0014)$. Comparison of large-diameter axons revealed a reverse trend, with a deficit of large axons in all CC segments of autistic subjects, ranging from $-34.02 \%$ in S I $(p<0.005)$ and $-58.2 \%$ in S V $(p<0.0001)$.

Table 5 summarizes the results of analyses focused on intersegmental differences in the ratio of axons with different diameters. In neurotypical subjects, a decrease in percentage of small-diameter axons was observed in all segments, but a significant decrease was detected in S III and SV. The increase in percentage of large-diameter axons was significant in all posterior segments in the control group. In the autistic group, the percentages of axons falling within each size range do not show a significant difference $(\mathrm{F}[6]=0.99, p=0.4310)$. Table 5 illustrates the absence of intersegmental differences in all CC segments in autistic subjects.

This pattern was the product of a significant developmental pathology of the CC of autistic subjects documented with an almost flat trendline in the percentage of small-, medium-, and large-diameter axons (Fig. 4). These flat trendlines distinguished the $\mathrm{CC}$ of autistic subjects from a very consistent and significant decrease in the percentage of small-diameter axons and consistent and significant increase in the percentage of large-diameter axons in the posterior segments of the $\mathrm{CC}$ of neurotypical subjects. The results reflected an almost total loss of structural and functional differentiation of individual segment connections critical for normal human brain function. 
Table 4 Different patterns of distribution of the percentage ( \pm SE) of small-, medium-, and large-diameter axons in segments I-V in autistic and control subjects

\begin{tabular}{|c|c|c|c|c|c|c|}
\hline \multirow[t]{2}{*}{ Axon diameter $(\mu \mathrm{m})$} & \multirow[t]{2}{*}{ Group } & \multicolumn{5}{|c|}{ Percentage of axons in CC segments } \\
\hline & & $\mathrm{SI}$ & $S \|$ & S III & S IV & SV \\
\hline \multirow[t]{4}{*}{$<0.651$} & C & $34.6(2.6)$ & $28.0(3.0)$ & $20.5(2.4)$ & $24.3(5.6)$ & $19.1(3.4)$ \\
\hline & A & $43.1(2.2)$ & $35.0(2.4)$ & $38.9(7.9)$ & $37.8(7.8)$ & $46.6(6.2)$ \\
\hline & $p<$ & $0.027^{*}$ & 0.093 & 0.058 & 0.175 & $0.0014^{* *}$ \\
\hline & Diff. & $+24.42 \%$ & ns & ns & ns & $+143.7 \%$ \\
\hline \multirow[t]{4}{*}{$0.651-1.051$} & C & $43.1(1.3)$ & $43.8(1.3)$ & $39.8(1.2)$ & $35.4(2.8)$ & $41.6(2.0)$ \\
\hline & A & $42.2(1.8)$ & $45.8(2.1)$ & $40.8(5.3)$ & $39.0(4.2)$ & $36.9(3.4)$ \\
\hline & $p<$ & 0.696 & 0.429 & 0.858 & 0.472 & 0.261 \\
\hline & Diff. & ns & ns & ns & ns & ns \\
\hline \multirow[t]{4}{*}{$>1.051$} & C & $22.2(1.9)$ & $28.2(2.1)$ & $39.6(3.3)$ & $40.2(3.8)$ & $39.2(3.2)$ \\
\hline & A & $14.7(1.3)$ & $19.0(2.1)$ & $20.3(3.0)$ & $23.1(3.8)$ & $16.4(3.1)$ \\
\hline & $p<$ & $0.005^{* *}$ & $0.008^{* *}$ & $0.007^{* * *}$ & $0.010^{* *}$ & $0.0001^{* * *}$ \\
\hline & Diff. & $-34.02 \%$ & $-32.52 \%$ & $-48.82 \%$ & -42.52 & $-58.2 \%$ \\
\hline
\end{tabular}

SE Standard error, CC corpus callosum, $S$ segment, $C$ control, $A$ autism; Diff., difference; ns, not significant. Standard errors in parentheses

The dominant role of axon number developmental deficits in a significant distortion of connectivity in all five CC segments in autistic subjects

The major factor defining structure and function of interhemispheric connectivity was the developmental focal and diffuse deficit of axons in all segments and in the entire $\mathrm{CC}$ of autistic subjects. The total number of myelinated axons was $49.42 \%$ less in the autistic (44.304 million) than in the control group (89.655 million) (Table 6).

The estimates of the total number of small-diameter $(<0.651 \mu \mathrm{m})$ axons revealed a significant reduction in $\mathrm{S}$ I and S III by 46.5 and $40.1 \%$, respectively (Table 7). Evaluation of the total number of large-diameter $(>1.05 \mu \mathrm{m})$ axons revealed a very consistent and significant deficit in all five segments in autistic subjects, by $72.6 \%$ on average. The number of medium-size axons (0.651 to $1.051 \mu \mathrm{m}$ in diameter) was also reduced significantly in all five segments, by $49.0 \%$ on average (Fig. 5 ).

Table 5 Significance of difference in the percentage of small-, medium-, and large-diameter axons in segment I compared to four other segments in the CC in autistic and control subjects

\begin{tabular}{llllll}
\hline Axon diameter $(\mu \mathrm{m})$ & Group & S I/S II & S I/S III & S I/S IV & S I/S V \\
\hline$<0.651$ & C & 0.073 & $0.002^{* *}$ & 0.073 & $0.002^{* *}$ \\
$0.651-1.051$ & C & 0.646 & 0.224 & 0.052 & 0.616 \\
$>1.051$ & C & $0.048^{*}$ & $0.0002^{* * *}$ & $0.0015^{* *}$ & $0.0002^{* * *}$ \\
$<0.651$ & A & $0.028^{*}$ & 0.663 & 0.663 & 0.663 \\
$0.651-1.051$ & $\mathrm{~A}$ & 0.232 & 0.745 & 0.404 & 0.232 \\
$>1.051$ & $\mathrm{~A}$ & 0.221 & 0.221 & 0.221 & 0.684 \\
\hline
\end{tabular}

$C C$ corpus callosum, $S$ segment, $C$ control, $A$ autism. $P$ values incorporate multiple-comparison adjustments to maintain a false discovery rate (FDR) of 0.05. ${ }^{*} p \leq 0.05 ;{ }^{* *} p \leq 0.01$; ${ }^{* * *} p \leq 0.001$
The study revealed multilevel consequences of two pathological developmental processes: those reducing the number of axons, and those reducing the diameter of axons in autistic subjects. The final product is a segment-specific deficit of small-, medium-, and largediameter axons, and loss of the integrity of interhemispheric connections in autistic subjects.

\section{Discussion}

Processing deficits in high-functioning autistic individuals are detected as shortages in integration of information and coordination of multiple neuronal networks $[35,36]$. The CC, the largest human brain commissure, provides long-range interhemispheric connections integrating and synchronizing anatomically and functionally specialized cortices. The pattern of detected pathology of $\mathrm{CC}$ connections in autistic subjects indicates that these abnormalities are a product of failure of two types of mechanisms: (a) those controlling the number of interhemispheric connections, and (b) those controlling axon structure, including axon diameter and cross-sectional area. Failure of the first type of mechanism results in loss of, or reduction in the number of axons and underconnectivity affecting all major anatomical and functional cortical regions. Failure of the second type of mechanisms results in reduction of axon diameter and cross-sectional area in all five CC segments already affected by an axon deficit. Functional studies have revealed that decrease of axon diameter correlates with reduction in the velocity and volume of signal transmission [8, 40, 47, 48]. Our study of autistic subjects provides evidence for the: (a) reduction in the number of long-range axonal connections in the $\mathrm{CC}$, (b) abnormal structure and function of preserved axons, 


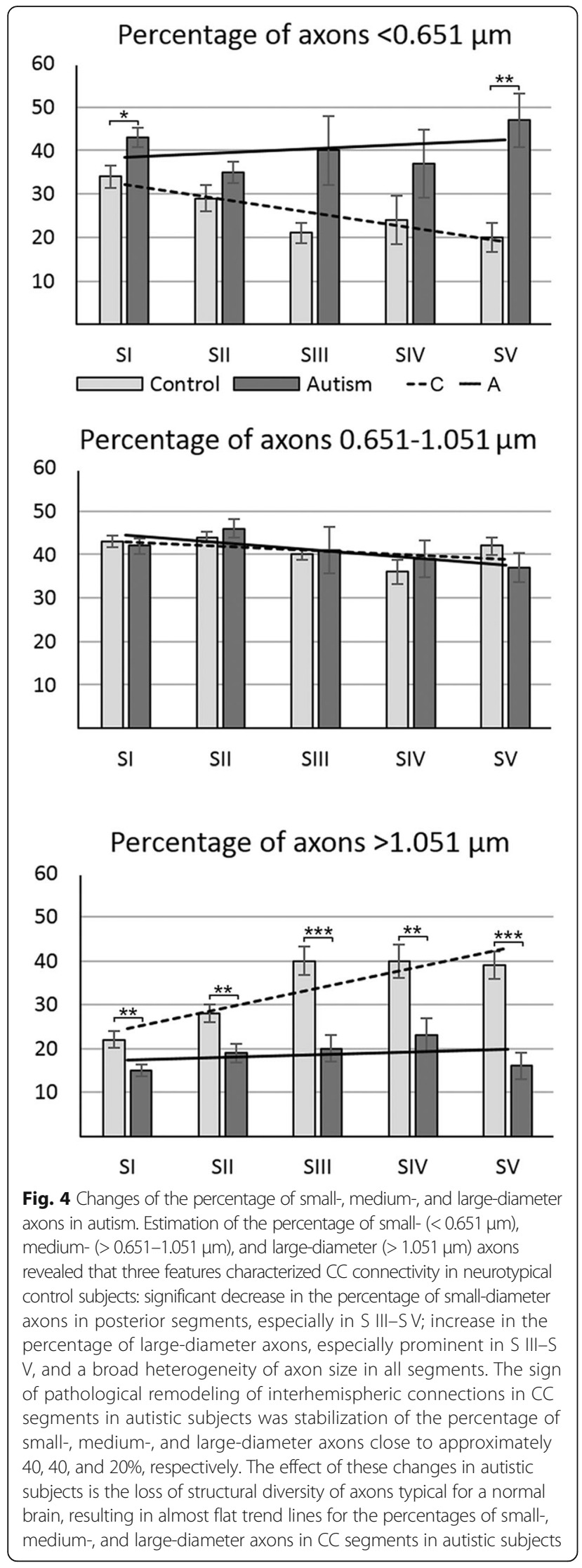

and (c) partial loss of structural and functional specialization of CC segments and integrity of interhemispheric connections.

\section{Interhemispheric underconnectivity}

Numerous structural MRI studies have revealed in autistic subjects a reduced size of the $\mathrm{CC}$, and abnormal shape, including thinning of the $\mathrm{CC}$, considered to be markers of abnormal interhemispheric connectivity $[10,14,20,21,27,29,41,50,54]$. The first postmortem study of 11 brains of autistic subjects, including nine brains examined in this ultrastructural study, expanded on MRI observations with light microscopy resolution and counts of axons in five CC segments [56]. In the examined brains of autistic subjects, two types of CC pathology contributed to interhemispheric underconnectivity: focal agenesis and diffuse hypoplasia. In three autistic subjects, total agenesis of S IV disconnected the sensory cortex, whereas partial agenesis of S III and S V partially disconnected the motor and parietal cortex. Partial agenesis of S I reduced interhemispheric connections of the prefrontal cortex. This topography revealed several CC segments with a high susceptibility to misguidance of axons and regional interhemispheric disconnection in autistic subjects. The second major cause of the deficit of interhemispheric connections detected in all examined autistic subjects was hypoplasia, defined by $\mathrm{CC}$ thinning, reduction of $\mathrm{CC}$ midsagittal area, and diffuse $62 \%$ reduction in the number of axons affecting all CC segments. The effect is loss of structural and functional integrity of interhemispheric connections, which may result in dysregulation of the velocity and volume of information transferred between hemispheres, loss of coherence and deficits in complex information processing in autistic subjects.

\section{Mechanisms contributing to underconnectivity and abnormal structure/function of axons}

Two independent and methodologically different studies of genes involved in neurodevelopmental disorders identified robust, statistically significant evidence for convergence of the input of ASD risk genes in glutamatergic projection neurons in cerebral cortex layers $2 / 3$ [37] and $5 / 6$ [59]. Neuropathological studies of cortical neurons revealed that the most common neuron developmental anomalies reported in the cerebral cortex of autistic subjects are multiregional reduction of neuron soma size $[26,52,53]$, minicolumn pathology always associated with a decrease in neuron size [11], and cortical dysplasia with smaller neurons [10, 57]. The link between reduced size of neurons and reduced axon diameter is strengthened by the fact that these reports were based 
Table 6 The difference between number (million \pm SE) of axons in five CC segments in autistic and control subjects

\begin{tabular}{lllllll}
\hline Group & SI & S II & S III & S IV & SV & Total number \\
\hline C & $25.900(1.891)$ & $24.944(1.175)$ & $9.878(1.417)$ & $3.711(0.326)$ & $25.222(2.518)$ & $89.655(4.186)$ \\
A & $10.933(2.010)$ & $15.500(1.642)$ & $3.211(0.644)$ & $1.360(0.441)$ & $13.300(1.618)$ & $44.304(4.885)$ \\
$p<$ & $0.0001^{* * *}$ & $0.0003^{* * *}$ & $0.0012^{* *}$ & $0.0006^{* * *}$ & $0.0011^{* *}$ & $0.0001^{* * *}$ \\
Differ. & $-57.79 \%$ & $-37.87 \%$ & $67.49 \%$ & $-63.35 \%$ & $-47.27 \%$ & $-49.42 \%$ \\
\hline
\end{tabular}

SE standard error, CC corpus callosum, $S$ segment, $C$ control, $A$ autism; Differ., difference. Standard errors in parentheses. ${ }^{* *} p \leq 0.01 ;{ }^{* * *} p \leq 0.001$

on examination of brains described in the current CC study. Energy capacity estimated by percentage of axon volume occupied with mitochondria increases with axon diameter [39]. One may assume that the energy capacity of smaller-diameter axons is proportional to the energy capacity of a reduced volume of neurons. CC connection pathology might be an effect of developmental anomalies of both neurons and glia. Long-range interhemispheric connectivity is controlled in utero by brain midline glial cells at six decision points. Misguidance of axons at any of these points by factors independent of the axon may change the trajectory and target of the axon [4, 43, 44] and result in partial CC agenesis and hypoplasia in autistic subjects.

\section{Broad range of differences in axon diameter in CC segments in neurotypical subjects}

Precision in synchronization of information transfer between hemispheres became more challenging with an evolutionary increase of brain size in apes and humans, which required both an increase in the velocity of long-distance interhemispheric conductivity and development of a variety of conduction velocities necessary for the functional diversity of human cortices and the brain [40].

Our study of neurotypical controls revealed a large range of axon diameters, reflecting a variety of conduction velocities, in each of the five CC segments, essential for cortices with different functions. Average axon diameter and cross-sectional area were the smallest in the first segment and increased in the posterior segments. The average diameter and cross-sectional area of axons in interhemispheric connections of the sensory cortex (S IV) were increased by 29 and $88 \%$, respectively, in comparison to axons of the prefrontal cortex (S I).

Another feature of the $\mathrm{CC}$ in neurotypical controls was an increase in the percentage of large-diameter $(>1.051 \mu \mathrm{m})$ axons, known as high-velocity axons, from $22 \%$ in connections of the prefrontal cortex (S I) to $40-39 \%$ in connections of the motor (S III), sensory (S IV), and parietal, occipital, and temporal cortices (S V). High-transmission velocity across distant regions is essential for synchronization of the activity of remote neuronal circuits [49]. The percentage of small-diameter axons $(<0.651 \mu \mathrm{m})$ decreased from 34 to $29 \%$ in S I and II to approximately $20-24 \%$ in $\mathrm{S}$ III-S V. The axon composition detected in the examined control group resembled a higher percentage of small-diameter axons in the anterior $\mathrm{CC}$ and a higher percentage of large-diameter axons in the posterior portion of the CC reported in other studies of neurotypical subjects. This pattern reflected the structural and functional specialization of each segment of

Table 7 The difference between number ( \pm SE) of small-, medium-, and large-diameter axons in five CC segments in autistic and control subjects

\begin{tabular}{|c|c|c|c|c|c|c|}
\hline Axon diameter & Group & SI & $S \|$ & S III & SIV & SV \\
\hline \multirow[t]{4}{*}{$<0.651$} & $C$ & $8.789(0.711)$ & $6.955(0.745)$ & $1.924(0.259)$ & $0.862(0.187)$ & $4.427(0.568)$ \\
\hline & A & $4.701(0.890)$ & $5.422(0.742)$ & $1.153(0.150)$ & $0.817(0.291)$ & $5.881(0.885)$ \\
\hline & $p<$ & 0.0025 & 0.164 (ns) & 0.0253 & 0.8916 (ns) & 0.1860 (ns) \\
\hline & Diff. & $-46.5 \%$ & & $-40.1 \%$ & & \\
\hline \multirow[t]{4}{*}{$>0.651<1.051$} & $C$ & $11.177(0.930)$ & $10.889(0.530)$ & 3.967 (0.639) & $1.319(0.144)$ & $10.405(0.934)$ \\
\hline & A & $4.704(0.972)$ & $7.083(0.836)$ & $1.633(0.332)$ & $0.757(0.178)$ & $5.084(0.974)$ \\
\hline & $p<$ & 0.0002 & 0.0014 & 0.0071 & 0.0294 & 0.0012 \\
\hline & Diff. & $-57.9 \%$ & $-35.0 \%$ & $-58.8 \%$ & $-42.6 \%$ & $-51.2 \%$ \\
\hline \multirow[t]{4}{*}{$>1.051$} & $C$ & $5.933(0.951)$ & 7.099 (0.735) & 3.985 (0.679) & $1.529(0.231)$ & $10.389(1.749)$ \\
\hline & A & $1.527(0.275)$ & $2.993(0.438)$ & $0.825(0.183)$ & $0.465(0.159)$ & $2.334(0.644)$ \\
\hline & $p<$ & 0.0015 & 0.0002 & 0.0014 & 0.0049 & 0.0015 \\
\hline & Diff. & $-74.2 \%$ & $-57.8 \%$ & $-79.3 \%$ & $-69.6 \%$ & $-77.6 \%$ \\
\hline
\end{tabular}

SE standard error, CC corpus callosum, $S$ segment, $C$ control, $A$ autism; Differ., difference. Standard errors in parentheses 


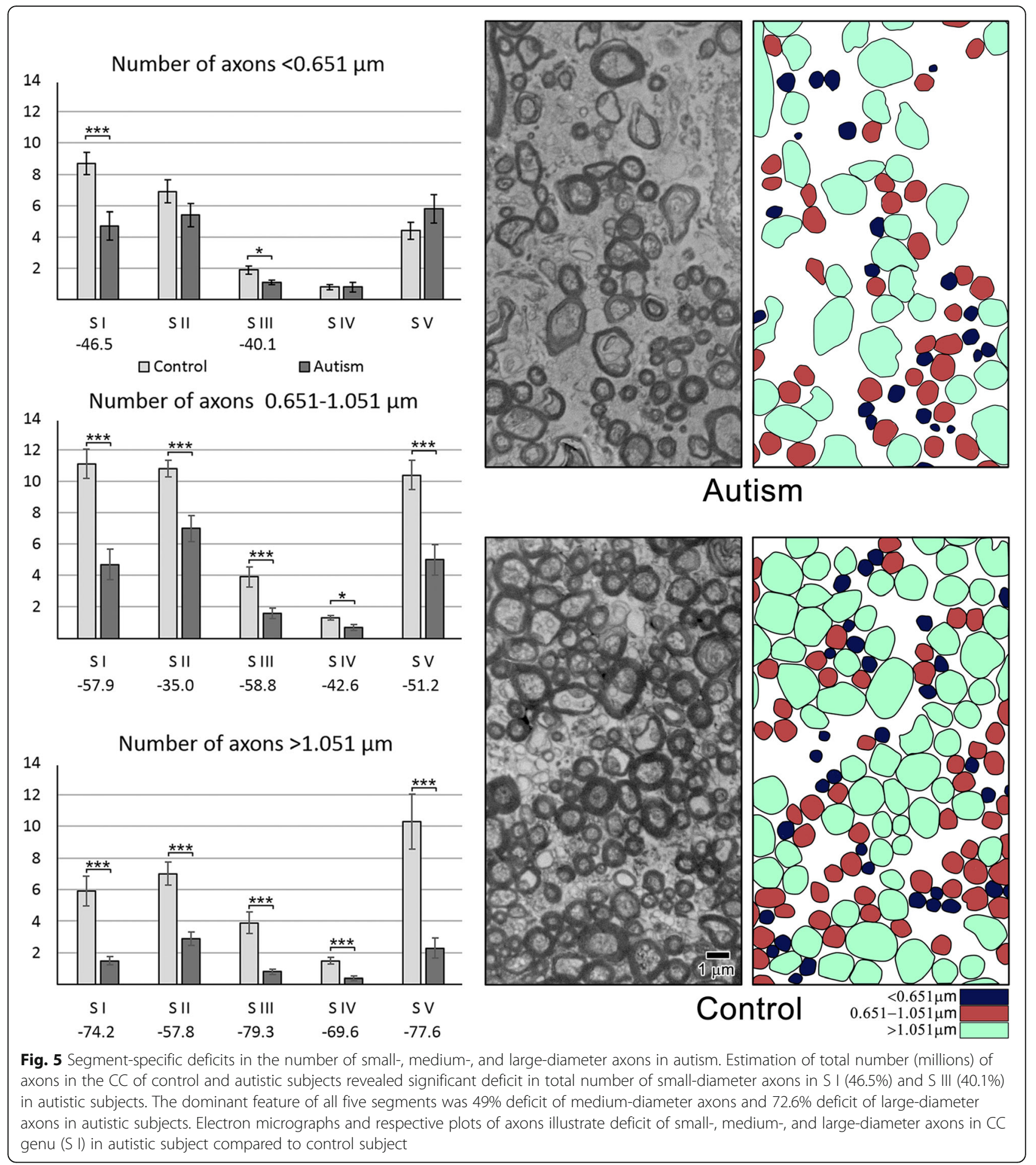

interhemispheric connections being critical for normal human brain function $[1,2,45]$.

\section{Loss of diversity of axonal connections in five CC} segments in autistic subjects

Velocity of signal transmission and firing rate correlate with axon diameter. Distribution of axon diameter, narrow or broad, symmetric or skewed, reflects the heterogeneity of information rates conveyed by the axon. A wide velocity range provides the wide range of timing necessary for synchronization of information delivery, processing, and response in an unaffected brain [39]. EM-based measurements of axons in autistic subjects revealed a reduction of average axon diameter by $12-29 \%$ 
and of average axon cross-sectional area by $25-52 \%$ in the rostro-caudal CC extent. These structural abnormalities resulted in a loss of the axon structural and functional diversification critical for regional and global cortical functionality.

Posterior CC segments are involved in the transfer of motor [55], somatosensory [13], and auditory and visual information [58] with a high demand for fast conduction and processing. However, reduction in the total number of axons with a diameter of $0.651-1.051 \mu \mathrm{m}$ by an average of $49 \%$, and of axons with a diameter $>1.051 \mu \mathrm{m}$ by an average of $72.6 \%$, is an indicator of a significant reduction in conductivity by both medium- and large-diameter axons. Moreover, the deficit of medium- and large-diameter axons affects not only posterior CC segments but also S I and S II connecting the prefrontal, premotor, and supplementary motor cortices. These deficits and the decreased diversification of interhemispheric connections may make a major contribution to the autism phenotype and comorbidities including intellectual deficits.

\section{Conclusions}

This study revealed the complex nature of developmental defects affecting the structure and function of interhemispheric connections in autistic subjects. Agenesis and hypoplasia caused a severe reduction in the number of CC axons and loss of integrity of interhemispheric connectivity. Developmental anomalies of axons included a significant increase in the percentage of small-diameter and low-velocity axons, and a decrease in the percentage of large-diameter high-velocity axons in the CC of autistic subjects. The loss of structural and functional diversity in $\mathrm{CC}$ segments in autistic subjects reflected a loss of specialization of interhemispheric connections essential for higher-order functions and for integration of multisensory perception. In general, light and electron microscopy studies indicate that autism is associated with extensive developmental distortion of structure and function of interhemispheric connectivity.

\begin{abstract}
Acknowledgments
Hemispheric brain samples were obtained from the Harvard Brain Tissue Resource Center, Belmont, MA, supported in part by PHS grant number R24$\mathrm{MH}$ 068855; the Brain and Tissue Bank for Developmental Disorders of the National Institute of Child Health and Human Development at the University at Maryland; and the New York State Brain and Tissue Bank at the Institute for Basic Research in Developmental Disabilities (IBR), Staten Island, NY. Tissue and clinical record acquisition was coordinated by the Autism Tissue Program (ATP; Director: Jane Pickett, Ph.D.) of Autism Speaks (AS) (currently Autism BrainNet, sponsored by the Simons Foundation and Autism Speaks). Tissue was processed, cut, and stained at IBR and shared with partnering institutions consistently with AS/ATP recommendations.

We thank Mrs. Maureen Marlow for manuscript editing.
\end{abstract}

\section{Funding}

This study was supported in part by funds from the New York State Office for People with Developmental Disabilities, a grant from the Department of Defense Autism Spectrum Disorders Research Program (AS073234, J.W., T.W.), and a grant from Autism Speaks (Princeton, NJ; J.W.).
Availability of data and materials

All data generated and analyzed during this study are included in the five tables in this article.

\section{Authors' contributions}

JW designed the project, drafted the manuscript, and together with WK performed the study. MF analyzed data. IK and KN assisted in tissue preservation and morphometric studies. VM-C. contributed to research design and writing manuscript. TW contributed to neuropathological evaluation and interpretation of clinicopathological data. JW. assisted in project design, coordination of tissue preservation and examination. All authors read and approved the final manuscript.

\section{Ethics approval and consent to participate}

All procedures involving human tissues were performed in accordance with the declaration of Helsinki. Experimental protocols were approved by IBR's Institutional Review Board.

\section{Consent for publication}

This postmortem study has been performed using anonymous coded brain tissue samples. Selected clinical records were extracted from the anonymous coded Autism Tissue Program - Autism Speaks database by an authorized project PI.

\section{Competing interests}

The authors declare that they have no competing interests.

\section{Publisher's Note}

Springer Nature remains neutral with regard to jurisdictional claims in published maps and institutional affiliations.

\section{Author details}

'Department of Developmental Neurobiology, New York State Institute for Basic Research in Developmental Disabilities, 1050 Forest Hill Road, Staten Island, NY 10314, USA. ${ }^{2}$ Research Design and Analysis Services, New York State Institute for Basic Research in Developmental Disabilities, Staten Island, NY 10314, USA. ${ }^{3}$ Pathology and Laboratory Medicine, Institute for Pediatric Regenerative Medicine, MIND Institute, University of California, Davis, CA, USA. ${ }^{4}$ Departments of Neurology, Pathology and Psychiatry, NYU Langone Medical Center, New York, NY, USA.

Received: 29 November 2018 Accepted: 2 December 2018

Published online: 19 December 2018

\section{References}

1. Aboitiz F, Montiel J (2003) one hundred million years of interhemispheric communication: the history of the corpus callosum. Braz J Med Biol Res 2003;36:409-420

2. Aboitiz F, Scheibel AB, Fisher RS, Zaidel E (1992) Fiber composition of the human corpus callosum. Brain Res 598:143-153

3. American Psychiatric Association (2013) Diagnostic and statistical manual of mental disorders, 5th edn. VA, Arlington

4. Bagri A, Marin O, Plump AS, Mak J, Pleasure SJ, Rubenstein JL et al (2002) Slit proteins prevent midline crossing and determine the dorsoventral position of major axonal pathways in mammalian forebrain. Neuron 33:233-248

5. Barnea-Goraly N, Kwon H, Menon V, Eliez S, Lotspeich L, Reiss AL (2004) White matter structure in autism: preliminary evidence from diffusion tensor imaging. Biol Psychiatry 55:323-326

6. Belmonte MK, Allen G, Beckel-Mitchener A, Boulanger LM, Carper RA, Webb SJ (2004) Autism and abnormal development of brain connectivity. J Neurosci 24:9228-9231

7. Benjamini Y, Hochberg Y (1995) Controlling the false discover rate: a practical and powerful approach to multiple testing. J Royal Statistical Society, Series B 57:289-300

8. Caminiti R, Carducci F, Piervincenzi C, Battaglia-Mayer A, Confalone G, Visco-Comandini F et al (2013) Diameter, length, speed, and conduction delay of callosal axons in macaque monkeys and humans: comparing data from histology and magnetic resonance imaging diffusion tractography. J Neurosci 33:14501-14511 
9. Casanova FM, El-Baz A, Elnakib A, Switala AE, Williams EL, Williams DL et al (2011) Quantitative analysis of the shape of the corpus callosum in autistic individuals. Autism 15:223-238

10. Casanova MF, El-Baz A, Kamat SS, Dombroski BA, Khalifa F, Elnakib A et al (2013) Focal cortical dysplasias in autism spectrum disorders. Acta Neuropathol Commun 1:67

11. Casanova MF, Van Kooten IAJ, Switala AE, Van Engeland $H$, Heinsen $H$, Steinbusch HWM et al (2006) Minicolumnar abnormalities in autism. Acta Neuropathol 112:287-303

12. Courchesne $\mathrm{E}$, Pierce $\mathrm{K}$ (2005) Why the frontal cortex in autism might be talking only to itself: local overconnectivity but long-distance disconnection. Curr Opin Neurobiol 23:225-230

13. Fabri M, Polonara G, Del Pesce M, Quattrini A, Salvolini U, Manzoni T (2001) Posterior corpus callosum and interhemispheric transfer of somatosensory information: an fMRI and neuropsychological study of a partially callosotomized patient. J Cogn Neurosci 13:1071-1079

14. Freitag $\mathrm{CM}$, Luders $\mathrm{E}$, Hulst HE, Narr KL, Thompson PM, Toga AW et al (2009) Total brain volume and corpus callosum size in medication-naive adolescents and young adults with autism spectrum disorder. Biol Psychiatry 66:316-319

15. Frith CD (2004) Is autism a disconnection disorder? Lancet Neurol 3:577

16. Geschwind DH, Levitt P (2007) Autism spectrum disorders: developmental disconnection syndromes. Curr Opin Neurobiol 17:103-111

17. Gleason JR. Within subjects (repeated measures) ANOVA, including between subjects factors. Stata Technical Bulletin 1999:47:40-45

18. Glickman ME, Rao SR, Schultz MR (2014) False discovery rate control is a recommended alternative to Bonferroni-type adjustments in health studies. J Clin Epidemiol 67:850-857

19. Greenhouse SW, Geisser S (1959) On methods in the analysis of profile data. Psychometrika 24:95-112

20. Hardan AY, Minshew NJ, Keshavan MS (2000) Corpus callosum size in autism. Neurology 55:1033-1036

21. Hardan AY, Pabalan M, Gupta N, Bansal R, Melhem NM, Fedorov S et al (2009) Corpus callosum volume in children with autism. Psychiatry Res Neuroimaging 174:57-61

22. Heinsen H, Arzberger T, Schmitz C (2000) Celloidin mounting (embedding without infiltration) - a new, simple and reliable method for producing serial sections of high thickness through complete human brains and its application to stereological and immunohistochemical investigation. J Chem Neuroanat 20:49-59

23. Highley JR, Esiri MM, McDonald B, Cortina-Borja M, Herron BM, Crow TJ (1999) The size and fiber composition of the corpus callosum with respect to gender and schizophrenia: a post-mortem study. Brain 122:99-110

24. Hofer S, Frahm J (2006) Topography of the human corpus callosum revisited-comprehensive fiber tractography using diffusion tensor magnetic resonance imaging. Neurolmage 32:989-994

25. Hughes JR (2007) Autism: the first firm finding = underconnectivity? Epilepsy Behav 11:20-24

26. Jacot-Descombes S, Uppal N, Wicinski B, Santos M, Schmeidler J, Giannakopoulos P et al (2012) Decreased pyramidal neuron size in Brodmann areas 44 and 45 in patients with autism. Acta Neuropathol 124:67-79

27. Just MA, Cherkassky VL, Keller TA, Kana RK, Minshew NJ (2007) Functional and anatomical cortical underconnectivity in autism: evidence from an fMRI study of an executive function task and corpus callosum morphometry. Cereb Cortex 17:951-961

28. Just MA, Cherkassky VL, Keller TA, Minshew NJ (2004) Cortical activation and synchronization during sentence comprehension in high-functioning autism: evidence of underconnectivity. Brain 127:1811-1821

29. Just MA, Keller TA, Malave VL, Kana RK, Varma S (2012) Autism as a neural systems disorder: a theory of frontal posterior underconnectivity. Neurosci Biobehav Rev 36:1292-1313

30. Kana RK, Keller TA, Minshew NJ, Just MA (2007) Inhibitory control in high functioning autism: decreased activation and underconnectivity in inhibition networks. Biol Psychiatry 62:198-206

31. Kumar A, Sundaram SK, Sivaswamy L, Behen ME, Makki MI, Ager J et al (2010) Alterations in frontal lobe tracts and corpus callosum in young children with autism spectrum disorder. Cereb Cortex 20:2103-2113

32. LaMantia AS, Rakic P (1990) Cytological and quantitative characteristics of four cerebral commissures in the rhesus monkey. J Comp Neurol 291:520-537
33. Lord C, Risi S, Lambrecht L, Cook EH Jr, Leventhal BL, DiLavore PC et al (2000) The autism diagnostic observation schedule-generic: a standard measure of social and communication deficits associated with the spectrum of autism. J Autism Dev Disord 30:205-223

34. Minshew NJ, Keller TA (2010) The nature of brain dysfunction in autism: functional brain imaging studies. Curr Opin Neurol 23:124-130

35. Minshew NJ, Sweeney J, Luna B (2002) Autism as a selective disorder of complex information processing and underdevelopment of neocortical systems. Mol Psychiatry 7(Suppl 2):S14-S15

36. Minshew NJ, Williams DL (2007) The new neurobiology of autism: cortex, connectivity, and neuronal organization. Arch Neurol 65:945-950

37. Parikshak NN, Luo R, Zhang A, Won H, Lowe JK, Chandran V et al (2013) Integrative functional genomic analyses implicate specific molecular pathways and circuits in autism. Cell 155:1008-1021

38. Paul LK, Van Lancker D, Schieffer B, Dietrich R, Brown WS (2003) Communicative deficits in individuals with agenesis of the corpus callosum: nonliteral language and affective prosody. Brain Lang 85:313-324

39. Perge JA, Niven JE, Mugnaini E, Balasubramanian V, Sterling P (2012) Why do axons differ in caliber? J Neurosci 32:626-638

40. Phillips KA, Stimpson CD, Smaers JB, Raghanti MA, Jacobs B, Popratiloff A et al (2015) The corpus callosum in primates: processing speed of axons and the evolution of hemispheric asymmetry. Proc R Soc B 282:1535

41. Piven J, Bailey J, Ranson BJ, Arndt S (1997) An MRI study of the corpus callosum in autism. Am J Psychiatry 154:1051-1056

42. Prigge MBD, Lange N, Bigler ED, Merkley TL, Neeley ES, Abildskov TJ et al (2013) Corpus callosum area in children and adults with autism. Res Autism Spectr Disord 7:221-234

43. Ren T, Anderson A, Shen W-B, Huang H, Plachez C, Zhang J et al (2006) Imaging, anatomical, molecular analysis of callosal formation in the developing human brain. Anat Record A 288A:191-204

44. Richards LJ, Plachez C, Ren T (2004) Mechanisms regulating the development of the corpus callosum and its agenesis in mouse and human. Clin Genet 66:276-289

45. Riise J, Pakkenberg B (2011) Stereological estimation of the total number of myelinated callosal fibers in human subjects. J Anat 218:277-284

46. Rippon G, Brock J, Brown C, Boucher J (2007) Disordered connectivity in the autistic brain: challenges for the "new psychophysiology". Int J Psychophysiol 63:164-172

47. Rushton WAH (1951) A theory on the effects of fibre size in medullated nerve. J Physiol 115:101-122

48. Seidl AH (2014) Regulation of conduction time along axons. Neuroscience 12:126-134

49. Singer W (1999) Neuronal synchrony: a versatile code for the definition of relations? Neuron:49-65

50. Stanfield AC, Mclntosh AM, Spencer MD, Philip R, Gaur S, Lawrie SM (2008) Towards a neuroanatomy of autism: a systematic review and meta-analysis of structural magnetic resonance imaging studies. Eur Psychiatry 23:289-299

51. StataCorp. Stata: Release 15. Statistical Software. College Station, TX: StataCorp LLC. 2017

52. Uppal N, Wicinski B, Buxbaum JD, Heinsen H, Schmitz C, Hof PR (2014) Neuropathology of the anterior midcingulate cortex in young children with autism. J Neuropathol Exp Neurol 73:891-902

53. van Kooten IAJ, Palmen SJMC, von Cappeln P, Steinbusch HWM, Korr H, Heinsen $\mathrm{H}$ et al (2008) Neurons in the fusiform gyrus are fewer and smaller in autism. Brain 131:987-999

54. Vidal CN, Nicolson R, DeVito TJ, Hayashi KM, Geaga JA, Drost DJ et al (2006) Mapping corpus callosum deficits in autism: an index of aberrant cortical connectivity. Biol Psychiatry 60:218-225

55. Wahl M, Lauterbach-Soon $B$, Hattingen $E$, Jung $P$, Singer $O$, Volz $S$ et al (2007) Human motor corpus callosum: topography, somatotopy, and link between microstructure and function. J Neurosci 27:12132-12138

56. Wegiel J, Flory M, Kaczmarski W, Brown WT, Chadman K, Wisniewski T et al (2017) Partial agenesis and hypoplasia of the corpus callosum in idiopathic autism. J Neuropathol Exp Neurol 76:225-237

57. Wegiel J, Kuchna I, Nowicki K, Imaki H, Wegiel J, Marchi E et al (2010) The neuropathology of autism: defects of neurogenesis and neuronal migration, and dysplastic changes. Acta Neuropathol 119:755-770

58. Westerhausen R, Grüner R, Specht K, Hugdahl K (2009) Functional relevance of interindividual differences in temporal lobe callosal pathways: a DTI tractography study. Cereb Cortex 19:1322-1329 
59. Willsey AJ, Sanders SJ, Li M, Dong S, Tebbenkamp AT, Muhle RA et al (2013) Coexpression networks implicate human midfetal deep cortical projection neurons in the pathogenesis of autism. Cell 155:997-1007

60. Witelson SF (1989) Hand and sex differences in the isthmus and genu of the human corpus callosum. A postmortem morphological study. Brain 112:799-835

61. Zikopoulos B, Barbas H (2010) Changes in prefrontal axons may disrupt the network in autism. J Neurosci 30:14595-14609

Ready to submit your research? Choose BMC and benefit from:

- fast, convenient online submission

- thorough peer review by experienced researchers in your field

- rapid publication on acceptance

- support for research data, including large and complex data types

- gold Open Access which fosters wider collaboration and increased citations

- maximum visibility for your research: over $100 \mathrm{M}$ website views per year

At BMC, research is always in progress.

Learn more biomedcentral.com/submissions 